\title{
PHOSPHORIC ACID AND SILICA OF PUERTO RICO SOILS
}

\author{
By JuAs AxTEDEe BoNinEI \\ Chief Soils Division
}

One hundred and nine soil samples from three areas in the humid region of Puerto Rico are grouped into five textural soil classes according to their mechanical composition, irrespective of their altitudes, and data are reported for $\mathrm{pH}$, total and available phosphoric acid and silica, supplemented also with some figures for voltume weight and altitude (tables 1-6).

Location and sampling of soils. The following three areas of . the humid region of Puerto Rico were chosen for this work:

1. Between the Arecibo and Manatí rivers including Utuado, Jayuya, Cialitos, Ciales, Florida, Arecibo and Barceloneta.

2. Between the Fajardo and Patillas rivers including Ceiba, Naguabo, Humacao, Las Piedras, Yabucoa, Maunabo and Patillas.

3. Around Caguas including Aguas Buenas, Gurabo, San Lorenzo and Las Cruces.

Soil survey maps were used for the location of the soil types. Samples to a depth of ten inches were taken with an auger in different places of the undisturbed field until a two-pound burlap bag was filled. The elevation of the field was read in a calibrated aneroid barometer.

Analytical methods. The analytical methods used were as follows:

\begin{tabular}{|c|c|}
\hline DETERMTINATION & MEthon \\
\hline $\begin{array}{l}\text { pH } \\
\text { Volume wright } \\
\text { Mechanical analysis } \\
\text { Total } \mathrm{P}_{5} \mathrm{O}_{5} \\
\text { Available } \mathrm{P}_{2} \mathrm{O}_{5} \text { and } \mathrm{SiO}_{2} \\
\text { Total } \mathrm{SiO}_{2}\end{array}$ & $\begin{array}{l}\text { Electrometric with quinhydrone } \\
\text { Cylinder (3) } \\
\text { Bouyoucos hydrometer (2) } \\
\text { Misnesium nitrate } \\
1 \% \text { Citric acid (1) } \\
\text { Sodium carbonate fusion }\end{array}$ \\
\hline
\end{tabular}

Available phosphoric acid and silice. were determined in the same aliquot.

Textural grades. The 109 soil types were classified according to their mechanical composition (4) using as a basis the Botyoucos system of soil separates: sands $(1.00-0.05 \mathrm{~mm}$.), silt $(0.05-0.005 \mathrm{~mm}$.), clay $(<0.005 \mathrm{~mm}$.). The soil types were grouped into five soil classes as follows: 3 sands (table 1), 19 sandy loams (table 2), 20 sandy clay loams (table 3), 14 clay loams (table 4 ) and 53 clays-1 sandy clay inclusive (table 5). The average values for volume weight, mechani- 
144 THE JOURNAL OP AGRICULTURE OF THE UNIVERSITY OF P. R.

TABLE 1. ACIDTTY, MECHANICAL ANATYSIS, TOTAL AND AVATLABLE PHOSPHORIC ACID AND SIIICA OF THREE SANDS IN THE HUMTD REGION OF PUERTO RICO

\begin{tabular}{|c|c|c|c|c|c|c|c|c|c|c|c|}
\hline \multirow{3}{*}{ Soil Type } & \multirow{3}{*}{\multicolumn{2}{|c|}{ Location }} & \multirow{3}{*}{$\begin{array}{l}\text { Elevation } \\
\text { (feet) }\end{array}$} & \multirow[b]{3}{*}{$\mathrm{pH}$} & \multicolumn{7}{|c|}{ Percentage on Dry Basis } \\
\hline & & & & & \multicolumn{3}{|c|}{ Mechanical Analysis } & \multicolumn{2}{|c|}{ Total } & \multicolumn{2}{|c|}{ Available } \\
\hline & & & & & Sand & Silt & Clay & $\mathrm{P}_{2} \mathrm{O}_{5}$ & $\mathrm{SiO}_{2}$ & $\mathrm{P}_{2} \mathrm{O}_{5}$ & $\overline{\mathrm{SiO}_{2}}$ \\
\hline Aguadilla sand & $\underset{\text { road }}{\mathrm{Km},} 72,0$ & Naguabo-Humacao & $\ldots$ & 6.5 & 89.0 & 3.2 & 7.8 & .051 & 74.61 & .003 & .028 \\
\hline Bayamón sand & $\underset{\text { road }}{\text { Km. }}$ & Arecibo-Barceloneta & 173 & 8.1 & 85.6 & 2.8 & 11.6 & .038 & 93.79 & .002 & .014 \\
\hline Bayamón sand & $\underset{\text { road }}{\mathrm{Km} .} 67.5$ & Arecibo-Barceloneta & $\cdots$ & 6.1 & 73.6 & 2.6 & 23.8 & .065 & 80.48 & .002 & .022 , \\
\hline
\end{tabular}

cal analysis, total and available phosphoric acid and silica are given in table 6. The weight of the soil calculated from the volume weight, and the ratio of total to available phosphoric acid are also reported in that table.

TAנLE 3. ACIDTTY, VOLUME WEIGHT, MECHANICAL, ANALYSIS, TOTAL AND AVAILABLE PHOSPHORIC ACID AND SIIICA OF NINETEEN SANDY LOAMS IN THE HUMID REGION OF PUERTO RICO

\begin{tabular}{|c|c|c|c|c|c|c|c|c|c|c|c|}
\hline \multirow[b]{3}{*}{ Soil Type } & \multirow[b]{3}{*}{ Location } & \multirow{3}{*}{$\begin{array}{l}\text { Elevation } \\
\text { (feet) }\end{array}$} & \multirow[b]{3}{*}{$\mathrm{pH}$} & \multirow{3}{*}{$\begin{array}{l}\text { Volyme } \\
\text { weight }\end{array}$} & \multicolumn{7}{|c|}{ Percentage on Dry Basis } \\
\hline & & & & & \multicolumn{3}{|c|}{ Mechanical Analysis } & \multicolumn{2}{|c|}{ Total } & \multicolumn{2}{|c|}{ Available } \\
\hline & & & & & Sand & Silt & Clay & $\mathrm{P}_{2} \mathrm{O}_{5}$ & $\mathrm{SiO}_{2}$ & $\mathrm{P}_{2} \mathrm{O}_{5}$ & $\mathrm{SiO}_{2}$ \\
\hline Caguas sandy & $\mathrm{Km} .58 .2 \quad$ Ceibs- & & & , & & . & & & . & & \\
\hline & outside of & 30 & 7.9 & 1.70 & 68.0 & 22.6 & 9.4 & .038 & 86.06 & .003 & .027 \\
\hline $\begin{array}{l}\text { Candelero sandy } \\
\text { loam }\end{array}$ & $\begin{array}{l}\text { Yabucoa playa road } \\
\text { about } 1.5 \mathrm{~km} \text {. from } \\
\text { main road }\end{array}$ & 53 & 5.5 & 1.58 & 72.4 & 14.0 & 13.6 & .057 & 70.01 & .003 & .034 \\
\hline $\begin{array}{l}\text { Candelero sandy } \\
\text { loam shallow } \\
\text { phase }\end{array}$ & $\begin{array}{l}\text { Yabucoa playa road } \\
\text { about } 1 \text { km. from } \\
\text { main road }\end{array}$ & 35 & 6.1 & 1.43 & 69.4 & 13.8 & 16.8 & .071 & 66.97 & .011 & .043 \\
\hline $\begin{array}{l}\text { Cayaguả sandy } \\
\text { loam }\end{array}$ & $\underset{\text { Patillas road }}{\mathrm{Km}, 1.8 \mathrm{San} \text { Iorenzo- }}$ & 480 & 5.3 & 1.48 & 58.8 & 24.4 & 16.8 & .117 & 62.05 & .041 & .153 \\
\hline $\begin{array}{l}\text { Ciales sandy } \\
\text { loam }\end{array}$ & $\begin{array}{c}\text { Colonia Ingenio, } \\
\text { Yabucoa, at Cerro } \\
\text { Santo Domingo }\end{array}$ & $\ldots$ & 5.7 & 1.30 & 74.8 & 12.0 & 13.2 & .107 & 59.33 & .027 & .051 \\
\hline $\begin{array}{l}\text { Daguno sundy } \\
\text { loam }\end{array}$ & $\underset{\text { IIatunabo road }}{\mathrm{Km}}$ & 117 & 6.4 & 1.47 & 70.2 & 12,4 & 17.4 & .124 & 49.18 & .012 & .146 \\
\hline $\begin{array}{l}\text { Maunabo sandy } \\
\text { loam }\end{array}$ & $\begin{array}{l}\text { Colonia Unión, Ya- } \\
\text { bucoa, } 1.5 \mathrm{~km} \text {. from } \\
\text { Yabucaa road at } \\
\text { Don Paco }\end{array}$ & $\cdots$ & 5.6 & 1.43 & 70,6 & 11.8 & 17.6 & .083 & 61.78 & .002 & .171 \\
\hline Pandura sandy & $\mathrm{Km} .103 .6$ Yabucoa- & 572 & 5.4 & 1.48 & 66.2 & 17.8 & 16.0 & .076 & 58.67 & .003 & .167 \\
\hline $\begin{array}{l}\text { Pandura sandy } \\
\text { loam }\end{array}$ & Km. 102.3 Yabucoa- & 368 & 5.6 & 1.30 & 78.4 & 11.6 & 10.0 & .147 & 62.31 & .027 & .098 \\
\hline $\begin{array}{l}\text { Pandura sandy } \\
\text { laam }\end{array}$ & $\begin{array}{l}\text { Km. } 107.3 \text { Yabucoa- } \\
\text { Maumabo road at } \\
\text { Barrio Talante }\end{array}$ & 358 & 6.0 & 1.29 & 70.4 & 15.8 & 13.8 & .158 & 53.89 & .031 & .130 \\
\hline $\begin{array}{l}\text { Pardura sandy } \\
\text { Loam }\end{array}$ & $\begin{array}{c}\mathrm{Km}, 101.0 \text { Yabucoa- } \\
\text { Miaunabo road }\end{array}$ & 162 & 5.7 & 1.46 & 66.0 & 15.6 & 18.4 & .062 & 63.07 & .002 & .088 \\
\hline Teja sendy loam & $\begin{array}{l}\text { Yabucoa playa road } \\
\text { about } 1 \mathrm{~km} \text {. from } \\
\text { main xoad }\end{array}$ & $\cdots$ & 5.6 & 1.40 & 68.0 & 19.6 & 12.4 & .088 & 58.61 & .002 & .054 \\
\hline Toa sandy loam & $\underset{\text { renzo-Patillas road }}{10.0}$ San & 600 & 5.3 & 1.39 & 62.8 & 22.4 & 14.8 & .085 & 55.30 & .006 & .108 \\
\hline
\end{tabular}


Table 2. Contintud

\begin{tabular}{|c|c|c|c|c|c|c|c|c|c|c|c|}
\hline \multirow[b]{3}{*}{ Soil Type } & \multirow{3}{*}{ Location } & \multirow{3}{*}{$\begin{array}{c}\text { Elevation } \\
\text { (feet) }\end{array}$} & \multirow{3}{*}{ pH } & \multirow{3}{*}{$\begin{array}{l}\text { Volume } \\
\text { weight }\end{array}$} & \multicolumn{7}{|c|}{ Percentage on Dry Basis } \\
\hline & & & & & \multicolumn{3}{|c|}{ Mechanical Analysis } & \multicolumn{2}{|c|}{ Total } & \multicolumn{2}{|c|}{ Available } \\
\hline & & & & & Send & Silt & Clay & $\mathrm{P}_{2} \mathrm{O}_{5}$ & $\mathrm{SiO}_{2}$ & $\mathrm{P}_{2} \mathrm{O}_{5}$ & $\mathrm{SiO}_{2}$ \\
\hline $\begin{array}{l}\text { Utuado sancy } \\
\text { loam }\end{array}$ & $\begin{array}{c}\text { About I/ } \mathrm{km} \text {. N. W. } \\
\text { Central Santa Bá- } \\
\text { bara, Jayuya }\end{array}$ & 1302 & 6.7 & $\cdots$ & 68.0 & 20.4 & 11.6 & .120 & 59.11 & .036 & .233 \\
\hline $\begin{array}{l}\text { Vega Alta sancy } \\
\text { loam }\end{array}$ & I/4 Km, W. Florida & 473 & 5.1 & & 77.8 & 7.8 & 14.4 & .120 & 84.24 & .006 & .020 \\
\hline Yivi sandy toam & $\begin{array}{l}\text { About } 1 / 2 \mathrm{~km} \text {. W. Cen- } \\
\text { trai Santa Bárbara, } \\
\text { Jayuya. } \\
\text { About } 1 / 2 \mathrm{~km} \text {. N. W. }\end{array}$ & 1202 & 5.6 & $\ldots$. & 56.0 & 28.8 & 15.2 & .179 & 54.54 & .038 & .300 \\
\hline Vivi sandy loam & $\begin{array}{l}\text { Central } \\
\text { Mfrunabo Colombia, } \\
\text { Ingerio road about .2 } \\
\mathrm{km} \text {. from Yabucoa }\end{array}$ & 39 & 5.5 & 1.35 & 58.4 & 21.8 & 19.8 & .155 & 58.10 & .019 & .200 \\
\hline & road & $\cdots$ & 6.9 & 1.52 & 74.8 & 12.0 & 13.2 & .085 & 63.96 & .012 & .089 \\
\hline $\begin{array}{l}\text { Yabucoa sandy } \\
\text { loam }\end{array}$ & $\begin{array}{c}\text { Colonia Ingenio, Ya- } \\
\text { bucoa }\end{array}$ & $\ldots$ & 5.0 & 1.53 & 74.4 & 10.4 & 15.2 & .140 & 67.04 & .037 & .049 \\
\hline
\end{tabular}

TABIE 3. ACIDITY, VOLUME WEIGHT, MECHANTCAL ANALXSIS, TOTAL AND AVAILABLE PHOSPHORIC ACID AND SIIICA OF TWENTY SANDY CLAY LOAMS IN THE HUMID REGION OF PUERTO RICO

\begin{tabular}{|c|c|c|c|c|c|c|c|c|c|c|c|}
\hline \multirow[b]{3}{*}{ Soil Type } & \multirow[b]{3}{*}{ Location } & \multirow{3}{*}{$\begin{array}{l}\text { Elevation } \\
\text { (feet) }\end{array}$} & \multirow[b]{3}{*}{$\mathrm{pH}$} & \multirow{3}{*}{$\begin{array}{l}\text { Volume } \\
\text { weight }\end{array}$} & \multicolumn{7}{|c|}{ Percentage on Dry Basis } \\
\hline & & & & & \multicolumn{3}{|c|}{ Mrechanical Analysis } & \multicolumn{2}{|c|}{ Total } & \multicolumn{2}{|c|}{ Available } \\
\hline & & & & & Sand & Silt & Clay & $\overline{\mathrm{P}_{2} \mathrm{O}_{5}}$ & $\mathrm{SiO}_{2}$ & $\mathrm{P}_{2} \mathrm{O}_{5}$ & $\mathrm{SiO}_{2}$ \\
\hline Alonso sandy & About $3 / 4 \mathrm{~km}$. S. W. & & & . & & $*$ & & & & , & \\
\hline & Humacao & 154 & 6.1 & 1.43 & 50.8 & 25.6 & 23.6 & .150 & 63.70 & .006 & .033 \\
\hline $\begin{array}{l}\text { Almirante sandy } \\
\text { clay loam }\end{array}$ & $\mathrm{r} / 2 \mathrm{~km}$. S. Florida & 484 & 5.0 & $\ldots$ & 53.2 & 18.0 & 28.8 & .289 & 59.89 & .012 & .022 \\
\hline $\begin{array}{l}\text { Cagras sandy } \\
\text { clay loam }\end{array}$ & Km. 1.0 Tomás de & 210 & 5.1 & 1.27 & 51.2 & 20.0 & 28.8 & .065 & 76.64 & .003 & .064 \\
\hline $\begin{array}{l}\text { Caguas sandy } \\
\text { clay loam }\end{array}$ & $\begin{array}{l}\text { Km. } 2.9 \text { Caguas-San } \\
\text { Lorenzo road }\end{array}$ & 90 & 4.3 & 1.40 & 55.2 & 20.0 & 24.8 & .064 & 78.00 & .007 & .063 \\
\hline $\begin{array}{l}\text { Cayaguá sandy } \\
\text { clay loam }\end{array}$ & $\begin{array}{l}\text { Km. } 1.8 \text { San Lorenza- } \\
\text { Patillas road }\end{array}$ & 480 & 5.3 & 1.48 & 58.8 & 24.4 & 16.8 & .117 & 62.05 & .041 & .153 \\
\hline $\begin{array}{l}\text { Cayaguá sandy } \\
\text { clay loam }\end{array}$ & $\begin{array}{l}\text { Km. } 9.0 \text { Caguas-San } \\
\text { Lorenzo road }\end{array}$ & 310 & 4.6 & 1.46 & 57.2 & 14.4 & 28.4 & .060 & 78.39 & .003 & .073 \\
\hline $\begin{array}{l}\text { Cayaguá sandy } \\
\text { clay loam }\end{array}$ & $\begin{array}{l}\mathrm{Km}, 25.2 \text { Las Piedras- } \\
\text { Humacao road }\end{array}$ & 210 & 4.9 & 1.59 & $51.2^{\circ}$ & 28.0 & 20.8 & .067 & 62.61 & .003 & .082 \\
\hline $\begin{array}{l}\text { Daguao sandy } \\
\text { clay loam }\end{array}$ & $\begin{array}{l}\mathrm{Km}, 109.0 \text { Yabucoa- } \\
\text { Mraunabo road, near } \\
\text { Maunabo }\end{array}$ & 39 & 6.7 & 1.42 & 58.0 & 15.8 & 26.2 & .135 & 53.42 & .007 & .218 \\
\hline $\begin{array}{l}\text { Hunacao sandy } \\
\text { clay loam }\end{array}$ & $\begin{array}{c}\mathrm{Km} .86 .0 \text { Humacao- } \\
\text { Yabucoa road op- } \\
\text { posite entrance to } \\
\text { Central Ejemplo }\end{array}$ & 117 & 6.1 & 1.16 & 50.4 & 26.0 & 23.6 & .135 & 65.14 & .015 & .083 \\
\hline $\begin{array}{l}\text { Las Piedras } \\
\text { sancy clay } \\
\text { loam }\end{array}$ & $\begin{array}{l}\text { Km. 24.1 Las Piedras- } \\
\text { Humacao road }\end{array}$ & 348 & 4.8 & 1.34 & 56.4 & 22.4 & 21.2 & .122 & 70.47 & .003 & .056 \\
\hline $\begin{array}{l}\text { Mariana sandy } \\
\text { clay loam }\end{array}$ & $\begin{array}{l}\text { Humacao-Antón Rriz } \\
\text { road at Barrio } \\
\text { Afambiche Blanco }\end{array}$ & 128 & 4.9 & 1.31 & 50.8 & 22.0 & 27.2 & .154 & 55.57 & .009 & .045 \\
\hline $\begin{array}{l}\text { Maunabo sandy } \\
\text { clay loam. }\end{array}$ & $\underset{\text { road }}{\mathrm{Km} .} 94.6 \mathrm{Yab}$ ucoa & $\cdots$ & 5.0 & 1.23 & 51.0 & 25.8 & 23.2 & .160 & 54.41 & .017 & .079 \\
\hline $\begin{array}{l}\text { Naranjito sandy } \\
\text { clay loatr }\end{array}$ & $\begin{array}{l}\mathrm{Km} \text {.113.5 Maunaba- } \\
\text { Patillas ro a d at } \\
\text { Cape Malapascua }\end{array}$ & 200 & 7.4 & 1.14 & 64.4 & 21.6 & 14.0 & .078 & 64.25 & .002 & .067 \\
\hline
\end{tabular}


TABLE 3. Continted

\begin{tabular}{|c|c|c|c|c|c|c|c|c|c|c|c|}
\hline \multirow[b]{3}{*}{ Soil Type } & \multirow[b]{3}{*}{ Location } & \multirow{3}{*}{$\begin{array}{c}\text { Elevation } \\
\text { (feet) }\end{array}$} & \multirow{3}{*}{$\mathrm{pH}$} & \multirow{3}{*}{$\begin{array}{l}\text { Volume } \\
\text { weight }\end{array}$} & \multicolumn{7}{|c|}{ Percentage on Dry Basis } \\
\hline & & & & & \multicolumn{3}{|c|}{ Arechanical Analysis } & \multicolumn{2}{|c|}{ Total } & \multicolumn{2}{|c|}{ Available } \\
\hline & & & & & Sand & Silt & Clay & $\mathrm{P}_{2} \mathrm{O}_{5}$ & $\mathrm{SiO}_{2}$ & $\overline{\mathrm{P}_{2} \mathrm{O}_{5}}$ & $\mathrm{SiO}_{2}$ \\
\hline $\begin{array}{l}\text { Pandura sandy } \\
\text { clay loam }\end{array}$ & $\begin{array}{l}\text { Km. } 104.8 \text { Yabucoa- } \\
\text { Maunabo road bor- } \\
\text { der line betw e } \mathrm{b} \\
\text { Barrio Pica, Ya. } \\
\text { hucoa and Barrio } \\
\text { Talante, Maunabo }\end{array}$ & 761 & 6.3 & 1.20 & 64.2 & 14.0 & 21.8 & .130 & 62.65 & .008 & .081 \\
\hline $\begin{array}{l}\text { Pandura sandy } \\
\text { clay loam }\end{array}$ & $\begin{array}{l}\mathrm{Km} \text { 105.8 Yabucoa. } \\
\text { Manuabo ro ad a t } \\
\text { Barrio Talante }\end{array}$ & 591 & 6.4 & 1.37 & 54.8 & 24.0 & 21.2 & .108 & 60.32 & .002 & .089 \\
\hline $\begin{array}{l}\text { Pandura snndy } \\
\text { clay loam }\end{array}$ & $\begin{array}{l}\text { Km. 7.0 San Lorenzo- } \\
\text { Patillas road }\end{array}$ & 500 & 5.3 & 1.40 & 58.8 & 14.4 & 26.8 & .116 & 62.43 & .005 & .117 \\
\hline $\begin{array}{l}\text { Riverwash } \\
\text { sandy clay } \\
\text { loam }\end{array}$ & $\begin{array}{l}\mathrm{Km} . \\
\text { road, Caguas }\end{array}$ & 440 & 5.3 & 1.39 & 54.0 & 22.4 & 23.6 & .194 & 63.68 & .016 & .139 \\
\hline $\begin{array}{l}\text { Sabana Secn } \\
\text { sandy clay } \\
\text { loam }\end{array}$ & $\begin{array}{l}\text { Between } \mathrm{km} .79 .3 \text { and } \\
79.4 \text { Arecibo- Barce- } \\
\text { loneta road }\end{array}$ & 17 & 8.2 & $\ldots$ & 60.2 & 11.8 & 28.0 & .133 & 73.62 & .004 & .055 \\
\hline $\begin{array}{l}\text { Talante sandy } \\
\text { clay loam }\end{array}$ & $\begin{array}{c}\text { About } 1 / 4 \text { km. Ya. } \\
\text { bucoa-Calabazas road }\end{array}$ & $\cdots$ & 5.2 & .98 & 53.0 & 20.2 & 26.8 & .141 & 53.81 & .004 & .162 \\
\hline $\begin{array}{l}\text { Teja sandy } \\
\text { clay loam }\end{array}$ & 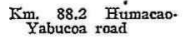 & • & 5.1 & 1.40 & 52.8 & 26.4 & 20.8 & .079 & 64.78 & .004 & .050 \\
\hline
\end{tabular}

TABLE 4. ACIDITY, VOLUME WEIGHT, MECHANICAL ANALYSIS, TOTAL AND AVAILABLE PHOSPHORIC ACID AND SILICA IN FOURTEEN CLAY LOAMS IN THE HUAIID REGION OF PUERTO RICO

\begin{tabular}{|c|c|c|c|c|c|c|c|c|c|c|c|}
\hline \multirow{3}{*}{ Soil Type } & \multirow[b]{3}{*}{ Location } & \multirow{3}{*}{$\begin{array}{l}\text { Elevation } \\
\text { (feet) }\end{array}$} & \multirow{3}{*}{$\mathrm{pH}$} & \multirow{3}{*}{$\begin{array}{l}\text { Volume } \\
\text { weight }\end{array}$} & \multicolumn{7}{|c|}{ Percentage on Dry Basis } \\
\hline & & & & & \multicolumn{3}{|c|}{ Nechanical Analysis } & \multicolumn{2}{|c|}{ Total } & \multicolumn{2}{|c|}{ Available } \\
\hline & & & & & Sand & Silt & Clay & $\mathrm{P}_{2} \mathrm{O}_{5}$ & $\mathrm{SiO}_{2}$ & $\mathrm{P}_{2} \mathrm{O}_{5}$ & $\mathrm{SiO}_{2}$ \\
\hline $\begin{array}{l}\text { Candelero clay } \\
\text { loam }\end{array}$ & $\underset{\text { Yabucos road }}{\mathrm{Km} .} 89.3 \underset{\text { Humaca- }}{\text { Had }}$ & 117 & 4.8 & 1.22 & 48.6 & 30.2 & 21,2 & .089 & 60.28 & .014 & .067 \\
\hline Coloso clay loam & $\begin{array}{l}\mathrm{Km}, 65.4 \text { Ceiba: } \\
\text { Naguabo road at } \\
\text { Qucbrada Palma }\end{array}$ & $\ldots$ & 6.1 & 1.32 & 48.8 & 23.6 & 27.6 & .115 & 65.01 & .005 & .151 \\
\hline Coloso clay loam & $\begin{array}{l}\text { Km } 0.8 \mathrm{Naguabo} \\
\text { Playa to Naguabo } \\
\text { road at Central } \\
\text { Triunfo }\end{array}$ & $\ldots$ & 5.4 & 1.34 & 26.6 & 44.2 & 29.2 & .138 & 60.22 & .007 & .239 \\
\hline $\begin{array}{l}\text { Dagueo clay } \\
\text { Joarm }\end{array}$ & $\begin{array}{l}\text { Entrance of road to } \\
\text { Centrat } \mathrm{Ejemplo} \\
\text { Humacso }\end{array}$ & 167 & 5.5 & 1.30 & 49.0 & 30.2 & 20.8 & .133 & 40.27 & .004 & .096 \\
\hline $\begin{array}{l}\text { Estación clay } \\
\text { lonm }\end{array}$ & $\mathrm{K}_{\text {Ciales road }} 11.9$ Manati- & 367 & 7.4 & .... & 48.0 & 26.4 & 25.6 & .224 & 53.61 & .029 & .656 \\
\hline$\underset{\text { Humacas }}{\text { Humay }}$ & $\begin{array}{l}\text { Entrance of road to } \\
\text { Central Ejemplo, } \\
\text { Humacao }\end{array}$ & 117 & 5.4 & 1.29 & 45.2 & 30.0 & 24.8 & .090 & 58.34 & .002 & .083 \\
\hline Juncos clay loam & $\begin{array}{l}\mathrm{Km} . \\
\text { Iorenzo road }\end{array}$ & 270 & 5.7 & 1.35 & 34.8 & 36.0 & 29.2 & .087 & 72.75 & .005 & .096 \\
\hline $\begin{array}{l}\text { Mariana clay } \\
\text { Ioam }\end{array}$ & 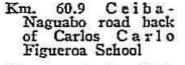 & 118 & 5.3 & 1.62 & $36.8^{\circ}$ & 38.0 & 25.2 & .018 & 74.93 & .002 & .277 \\
\hline $\begin{array}{l}\text { Rio Arriba clay } \\
\text { loam }\end{array}$ & $\begin{array}{l}\text { Humacao-Antón Ruiz } \\
\text { road at Ba r i io } \\
\text { NTambiche Blanco }\end{array}$ & 136 & 6.1 & 1.41 & 41.6 & 30.4 & 28.0 & .167 & 63.84 & .003 & .045 \\
\hline
\end{tabular}


TABle 4. Continued

\begin{tabular}{|c|c|c|c|c|c|c|c|c|c|c|c|}
\hline \multirow[b]{3}{*}{ Soil Type } & & \multirow{3}{*}{$\begin{array}{c}\text { Elevation } \\
\text { (feet) }\end{array}$} & \multirow{3}{*}{$\mathrm{pH}$} & \multirow{3}{*}{$\begin{array}{l}\text { Volume } \\
\text { weight }\end{array}$} & \\
\hline & & & & & \multicolumn{3}{|c|}{ Mechanical Analysis } & \multicolumn{2}{|c|}{ Total } & \multicolumn{2}{|c|}{ Available } \\
\hline & Location & & & & Sand & Silt & Clay & $\overline{\mathrm{P}_{2} \mathrm{O}_{5}}$ & $\overline{\mathrm{SiO}_{2}}$ & $\mathrm{P}_{2} \mathrm{O}_{5}$ & $\mathrm{SiO}_{2}$ \\
\hline $\begin{array}{l}\text { Sabana clay } \\
\text { loam }\end{array}$ & $\begin{array}{l}\text { Km. } 112.8 \text { Nraunabo } \\
\text { Patillas road }\end{array}$ & 130 & 7.1 & 1.10 & 48.4 & 24.2 & 27.4 & .078 & 56.15 & .003 & .101 \\
\hline Toa clay loam & Km. 0.1 Tomás de & 180 & 5.9 & 1.18 & 48.0 & 24.0 & 28.0 & .137 & 62.50 & .011 & .174 \\
\hline Toa clay loam & Km. 1.5 Caguas-San & 80 & 5.8 & 1.40 & 50.8 & 24.0 & 25.2 & .119 & 63.70 & .007 & .176 \\
\hline Via clay loam & $\begin{array}{l}\text { Humacao-Antón Ruiz } \\
\text { road at Barrio } \\
\text { Mambiche Blanco }\end{array}$ & 109 & 6.4 & 1.38 & 42.6 & 29.4 & 28,0 & .135 & 68.14 & .003 & .076 \\
\hline Via clay loam & $\begin{array}{l}\mathrm{Km} .0 .7 \text { Humacao- } \\
\text { Antón Ruiz road at } \\
\text { Perseverancia farm }\end{array}$ & 107 & 6.4 & 1.09 & 41.2 & 24.8 & 34.0 & .100 & 54.01 & .002 & .093 \\
\hline
\end{tabular}

TABLE 5. ACIDITY, VOLUMIE WEIGHT, MIECHANICAL ANALYS1S, TOTAL AND AVAILABILE PHOSPHORIC ACID AND SILICA, OF' FIFTY-THREE CLAYS IN THE HUNID REGION OF PUERTO RICO

\begin{tabular}{|c|c|c|c|c|c|c|c|c|c|c|c|}
\hline \multirow{3}{*}{ Soil Type * } & \multirow[b]{3}{*}{ Lacation } & \multirow{3}{*}{$\underset{\text { Elevation }}{\text { (feet) }}$} & & \multirow{3}{*}{$\begin{array}{l}\text { Volume } \\
\text { weight }\end{array}$} & \\
\hline & & & & & \multicolumn{3}{|c|}{ Mechanieal Analysis } & \multicolumn{2}{|c|}{ Total } & \multicolumn{2}{|c|}{ Available } \\
\hline & & & & & Sand & Siit. & Clay & $\mathrm{P}_{2} \mathrm{O}_{5}$ & $\mathrm{SiO}_{2}$ & $\mathrm{P}_{2} \mathrm{O}_{5}$ & $\mathrm{SiO}_{2}$ \\
\hline Alonso clay & $\begin{array}{c}\text { About } \mathrm{km}, 19.5 \text { Ciales- } \\
\text { Jayuya road }\end{array}$ & 1034 & 5.4 & $\cdots$ & 28.2 & 33.0 & 38.8 & .068 & 48.63 & .002 & .080 \\
\hline $\begin{array}{l}\text { Alonso clay } \\
\text { smooth phase }\end{array}$ & $\begin{array}{l}\text { About } \mathrm{km}, 21.2 \text { Flor- } \\
\text { ida-Jayuya road at } \\
\text { Barrio Piedra Gorda } \\
\text { School }\end{array}$ & 759 & 4.7 & $\ldots$ & 26.6 & 28.0 & 45.4 & .065 & 66.35 & .001 & .044 \\
\hline Caguas clay & $\underset{\text { Cayey road }}{\mathrm{Km}} 37.7 \mathrm{C}$ u a s- & 280 & 6.5 & & 31.2 & 32.8 & 36.0 & .113 & 76.49 & .003 & .074 \\
\hline Caguas clay & $\underset{\text { Patilias road }}{\text { Km. }}$ & 620 & 5.2 & 1.46 & 42.8 & 20.4 & 36.8 & .089 & 67.44 & .001 & .065 \\
\hline Cayaguá clay & $\begin{array}{l}\mathrm{Km} .5 .7 \text { San Iorenzo- } \\
\text { Patillas road at en. } \\
\text { trance of } \mathrm{J} \text { a g u a } \mathrm{r} \\
\text { road }\end{array}$ & 600 & 5.7 & 1.25 & 24.8 & 34.4 & $\dot{1}_{40.8}$ & .106 & 72.38 & .006 & .202 \\
\hline Catalina clay & $\begin{array}{l}\text { About km. } 18.5 \text { Ciales- } \\
\text { Jayusa road }\end{array}$ & 940 & 5.1 & ... & 32.0 & 24.0 & 44.0 & .073 & 56.09 & .004 & .036 \\
\hline $\begin{array}{l}\text { Catalina clay } \\
\text { steep phase }\end{array}$ & $\begin{array}{l}0.5 \mathrm{~km} . \text { E. Hacienda } \\
\text { Pjecira Gorda, Flor- } \\
\text { ida-Jaynya road }\end{array}$ & 685 & 5.1 & . & 35.6 & 22.4 & 42.0 & .139 & 45.71 & .012 & .077 \\
\hline Ciales clay & $\underset{\text { Jayuya road }}{15.0} \mathrm{Cia}$ es & 639 & 4.6 & . & 25.2 & 28.4 & 46.4 & .132 & 48.71 & .008 & .068 \\
\hline Ciales clay & $\mathrm{Km}_{\text {Ciales road }} 12.5$ Manati- & 367 & 7.6 & & 35.6 & 26.4 & 38.0 & .153 & 61.35 & .012 & .131 \\
\hline Cialitos clay & Km, ${ }_{\text {Cayey road }}^{47.9 ~} \mathrm{C}$ a g u s - & 1525 & 4.5 & 1.24 & 23.6 & 34.0 & 42.4 & .184 & 70.60 & .002 & .025 \\
\hline Cialitos clay & $\mathrm{K}_{\text {Cayey road }} 45.5 \mathrm{C}$ a $\mathrm{g}$ as - & 1200 & 4.2 & 1.42 & 35.6 & 24.0 & 40.4 & .169 & 69.88 & .002 & .026 \\
\hline Cialitos clay & $\begin{array}{l}\text { About } 1 \text { km, S. E. } \\
\text { Haciende Pi edra } \\
\text { Gorda, Flor i da- } \\
\text { Jayuya road }\end{array}$ & 892 & 4.9 & $\ldots \therefore$ & 29.8 & 32.8 & 37.4 & .084 & 57.50 & .005 & .410 \\
\hline Cialitos clay & $\begin{array}{c}\text { Between } \mathrm{km} .20 .2-20.3 \\
\text { Florida-Jayuya rond }\end{array}$ & 685 & 5.8 & & 35.6 & 30.4 & 34.0 & .056 & 61.68 & .014 & .219 \\
\hline $\begin{array}{l}\text { Cialitos clay } \\
\text { steep phase }\end{array}$ & $\begin{array}{l}\text { Camino Borinquen at } \\
\text { km. } 5.3 \text { Naranjito } \\
\text { road }\end{array}$ & 700 & 5.2 & 1.47 & 40.0 & 28.4 & 31.6 & .152 & 62.16 & .006 & .157 \\
\hline
\end{tabular}


148 THE JOURNAL OF AGRICULTURE OF THE UNIVERSITY OF P. R.

TABLE 5. Continted

\begin{tabular}{|c|c|c|c|c|c|c|c|c|c|c|c|}
\hline \multirow[b]{3}{*}{ Soil Type } & \multirow[b]{3}{*}{ Location } & \multirow{3}{*}{$\begin{array}{l}\text { Elevation } \\
\text { (feet) }\end{array}$} & \multirow{3}{*}{$\mathrm{pH}$} & \multirow{3}{*}{$\begin{array}{l}\text { Volume } \\
\text { weight }\end{array}$} & \multicolumn{7}{|c|}{ Percentage on Dry Basis } \\
\hline & & & & & \multicolumn{3}{|c|}{ Mechanical Analysis } & \multicolumn{2}{|c|}{ Total } & \multicolumn{2}{|c|}{ Available } \\
\hline & & & & & Sand & Silt & Clay & $\mathrm{P}_{3} \mathrm{O}_{5}$ & $\mathrm{SiO}_{2}$ & $\mathrm{P}_{2} \mathrm{O}_{5}$ & $\mathrm{SiO}_{2}$ \\
\hline Coloso clay & $\begin{array}{l}\mathrm{Km} \text { 0.5 Humacao: } \\
\text { Antón Rutiz road at } \\
\text { Perseverancia farm }\end{array}$ & 107 & 7.4 & 1.24 & 34.4 & 10.0 & 55.6 & .121 & $\$ 1.71$ & .006 & .124 \\
\hline Coloso clay & $\begin{array}{l}\text { Km. } 112.2 \text { Míaunabo- } \\
\text { Patillas road }\end{array}$ & 39 & 8.1 & 1.20 & 38.8 & 28.0 & 33.2 & .088 & 59.63 & .011 & .173 \\
\hline Coloso clay & $\begin{array}{l}\text { About } 3 / 4 \mathrm{~km} \text {. S. W. } \\
\text { Cent ra } 1 \text { Plazuela, } \\
\text { Barceloneta }\end{array}$ & 23 & 7.0 & $\cdots \cdots$ & 24.0 & 32.8 & 43.2 & .216 & 53.05 & .014 & .484 \\
\hline Coloso clay & 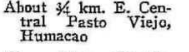 & 16 & 4.9 & 1.15 & 38.6 & 27.8 & 33.6 & .244 & 62.25 & .018 & .048 \\
\hline Coto clay & 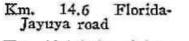 & 723 & 8.2 & $\cdots$ & 42.8 & 24.0 & 33.2 & .460 & 26.83 & .017 & .051 \\
\hline Coto day & $\begin{array}{l}\text { Km. } 12.1 \text { Fior ida - } \\
\text { Jayuya road }\end{array}$ & 555 & 8.1 & $\cdots$ & 21.0 & 20.8 & 58.2 & .525 & 39.36 & .017 & .044 \\
\hline Daguao clay & $\begin{array}{l}\text { Km. } 65.0 \text { Ceiba- } \\
\text { Naguabo road at } \\
\text { Colonia Esperanza } \\
\text { Barrio Daguao }\end{array}$ & 116 & 6.3 & 1.40 & 24.4 & 30.4 & 45.2 & .056 & 49.26 & .003 & .060 \\
\hline Espinosa clay & $\begin{array}{c}\text { Km. } \quad 78.1 \text { Ärecibo- } \\
\text { Barceloneta road }\end{array}$ & 90 & 7.4 & $\cdots$ & 50.0 & 10.8 & 39.2 & .119 & 64.16 & .002 & .064 \\
\hline Espinosa clay & $\underset{\text { Barceloneta }}{74.8} \underset{\text { road }}{\text { Arecibo- }}$ & $\cdots \cdots$ & 6.1 & $\cdots$ & 51.4 & 11.4 & 37.2 & .086 & 67.27 & .002 & .035 \\
\hline Irurena clay & $\begin{array}{l}\text { Colonia Jnión about } \\
2 \mathrm{~km} \text {. from } \mathrm{Y} \text { abucoa } \\
\text { road at Moquilla }\end{array}$ & $\cdots$ & 6.1 & 1.15 & 44.2 & 23.8 & 32.0 & .162 & 53.88 & .009 &. .235 \\
\hline Juncos clay & $\begin{array}{l}\text { About I km. S. E. } \\
\text { Hacienda P i ed ra } \\
\text { Gor d a, FIorida- } \\
\text { Jayuya road }\end{array}$ & 891 & 5.9 & $\cdots$ & 47.0 & 24.2 & 28.8 & .057 & 52.95 & .004 & .886 \\
\hline Juncos clay & $\begin{array}{l}\text { Borinquen road, Ca- } \\
\text { guas, about } 0.6 \mathrm{~km} \text {. } \\
\text { Naranjito road. }\end{array}$ & 525 & 5.6 & 1.28 & 26.0 & 32.4 & 41.6 & .113 & 69.02 & .003 & .072 \\
\hline Juncos clay & $\underset{\text { Cayey road }}{\mathrm{Km} .} 40.3 \mathrm{Ca}$ a - & 390 & 6.5 & 1.34 & 27.2 & 28.8 & 44.0 & .106 & 56.49 & .002 & .174 \\
\hline Juncos clay & $\begin{array}{l}\text { Km. } 1.6 \text { Caguas-Tomás } \\
\text { de Castro road }\end{array}$ & 280 & 6.0 & 1.36 & 26.8 & 34.0 & 39.2 & .073 & 58.51 & .004 & .166 \\
\hline Juncos clay & $\begin{array}{c}\text { Km. } 8.0 \text { Caguas-San } \\
\text { Lorenzo rosd }\end{array}$ & 280 & 6.3 & 1.24 & 28.8 & 30.8 & 40.4 & .118 & 66.43 & .006 & .175 \\
\hline Las Piedras clay & $\begin{array}{c}\text { Km. } 2.0 \text { Jaguar road, } \\
\text { San Lorenzo }\end{array}$ & 700 & 6.2 & 1.05 & 38.8 & 30.4 & 30.8 & .155 & 66.93 & .004 & .099 \\
\hline Las Piedras elay & Km. 6.0 Caguas-San & 275 & 6.7 & 1.07 & 30.4 & 28.8 & 40.8 & .142 & 62.25 & .007 & .169 \\
\hline Los Guineos clay & $\begin{array}{l}\text { Km. } 27.8 \text { Casa Blanca- } \\
\text { Jayuya road }\end{array}$ & 2134 & 5.0 & $\cdots \cdots$ & 31.8 & 37.4 & 30.8 & .187 & 34.77 & .005 & .065 \\
\hline Los Guineos clay & $\underset{\text { Jayuya road }}{\text { Kma }} 30.9 \mathrm{Ci}$ es - & 1679 & 4.5 & $\cdots$ & 29.2 & 24,0 & 46.8 & .141 & 35.29 & .001 & .061 \\
\hline Múcara clay & $\begin{array}{l}\text { Km, } 5.7 \\
\text { Tomás de } \\
\text { road }\end{array}$ & 870 & 5.5 & 1.29 & 28.8 & 28.4 & 42.8 & .095 & 57.07 & .003 & .154 \\
\hline Múcaxa clay & $\underset{\text { Cayey road }}{\mathrm{Km}} 43.1 \mathrm{Cag}$ - & 770 & 5.1 & 1,35 & 43.6 & 26.8 & 29.6 & .097 & 53.09 & .003 & .094 \\
\hline MIúcara clay & $\begin{array}{l}\text { Borinquen road, Ca- } \\
\text { guas, at km. } 3.8 \\
\text { camino Naranjito }\end{array}$ & 680 & 5.7 & 1.07 & 31.6 & 30.4 & 38.0 & .148 & 61.61 & .001 & .173 \\
\hline Múcara clay & $\begin{array}{l}\text { Km. } 3.0 \text { Caguas- } \\
\text { Tomás de Castro. } \\
\text { road }\end{array}$ & 600 & 5.7 & 1.27 & 30.8 & 32,0 & 37.2 & .091 & 52.90 & .004 & .181 \\
\hline
\end{tabular}


TABLE 5. Continued

\begin{tabular}{|c|c|c|c|c|c|c|c|c|c|c|c|}
\hline \multirow[b]{3}{*}{ Soil Type } & \multirow[b]{3}{*}{ Location } & \multirow{3}{*}{$\begin{array}{c}\text { Elevation } \\
\text { (feet) }\end{array}$} & \multirow{3}{*}{$\mathrm{pH}$} & \multirow{3}{*}{$\begin{array}{l}\text { Volume } \\
\text { weight }\end{array}$} & \multicolumn{7}{|c|}{ Percentage on Dry Basis } \\
\hline & & & & & \multicolumn{3}{|c|}{ Mrechanical Analysis } & \multicolumn{2}{|c|}{ Total } & \multicolumn{2}{|c|}{ Available } \\
\hline & & & & & Sand & Silt & Clay & $\mathrm{P}_{2} \mathrm{O}_{5}$ & $\mathrm{SiO}_{2}$ & $\mathrm{P}_{2} \mathrm{O}_{5}$ & $\mathrm{SiO}_{2}$ \\
\hline Mrúcara clay & $\begin{array}{c}\mathrm{Km} .42 .0 \mathrm{Cag} \text { a a s- } \\
\text { Cayey road at en- } \\
\text { trance of of Camino } \\
\text { Borinquen }\end{array}$ & 510 & 5.2 & 1.48 & 35.6 & 26.8 & 37.6 & .201 & 54.18 & .003 & .155 \\
\hline Niúcata clay & $\begin{array}{c}\text { Km. } 7,0 \text { Caguas- } \\
\text { Tomás de Castro } \\
\text { road }\end{array}$ & 280 & 5.2 & 1.07 & 34.8 & 26.4 & 38.8 & .144 & 56.79 & .005 & .107 \\
\hline $\begin{array}{l}\text { Mícara clay } \\
\text { steep phase }\end{array}$ & $\underset{\text { Naguabo road }}{\mathrm{Km}} \quad 68.0 \mathrm{Ceiba}-$ & 80 & 6.1 & 1.15 & 36.4 & 4.8 & 58.8 & .053 & 53.11 & .002 & .123 \\
\hline $\begin{array}{l}\text { Mrúcare sandy } \\
\text { clay }\end{array}$ & $\begin{array}{l}\mathrm{Km} 1.2 \text { Fumacao- } \\
\text { Antón Rtiz road, at } \\
\text { Barrio Pitijalla }\end{array}$ & 173 & 6.3 & 1.17 & 53.2 & 12.8 & 34.0 & .071 & 49.94 & .002 & .091 \\
\hline Niaranjito clay & $\begin{array}{c}\text { Borinquen road, Ca- } \\
\text { guas, at km. } \\
\text { Naranjito road }\end{array}$ & 620 & 5.1 & 1.14 & 29.6 & 26.4 & 44.0 & .087 & 60.70 & .001 & .086 \\
\hline Reparada clay & $\begin{array}{l}\mathrm{Km} \text {. } 78.7 \text { Naguabo } \\
\text { road at Colonia } \\
\text { Santa Texesa }\end{array}$ & $\ldots$ & 4.7 & .43 & 48.8 & 20.4 & 30.8 & .233 & 41.34 & .034 & .086 \\
\hline Río Piedras clay & $\begin{array}{l}\text { About } 21.2 \text { Florida- } \\
\text { Jayuya road at } \\
\text { Barrio Piedra Gorda } \\
\text { School }\end{array}$ & 762 & 6.3 & $\cdots$ & 33.6 & 27.6 & 38.8 & .068 & 53.71 & .002 & .057 \\
\hline Sabana clay & $\begin{array}{l}\mathrm{Km} .71 .4 \text { Naguabo- } \\
\text { Humacao road }\end{array}$ & 70 & 4.5 & 1.33 & 46.4 & 22.0 & 31.6 & .128 & 64.64 & .004 & .032 \\
\hline Sabana Seca clay & $\underset{\text { road, Caguas }}{\text { Km. }}$ & 480 & 6.2 & 1.32 & 27.2 & 30.8 & 42.0 & .112 & 58.97 & .003 & .140 \\
\hline Toa clay & $\begin{array}{l}\text { Km. } 1.3 \text { Jagraar road, } \\
\text { Sar Lotenzo }\end{array}$ & 590 & 5.7 & 1.08 & 24.8 & 38.4 & 36.8 & .106 & 57.35 & .005 & .421 \\
\hline Toa clay & $\mathrm{Km}_{\text {Cayey road }} 41.75$ Caguas- & 420 & 5.3 & 1.36 & 45.2 & 21.2 & 33.6 & .164 & 58.92 & .005 & .200 \\
\hline Toz clay & $\begin{array}{l}\mathrm{Km} \text {, } 1.0 \text { Borinquen } \\
\text { roagus }\end{array}$ & 420 & 5.8 & 1.24 & 31.6 & 28.4 & 40.0 & .099 & 63.16 & .008 & .109 \\
\hline Toa clay & $\begin{array}{l}\text { About } 1 / 2 \mathrm{~km} . \mathrm{E} . \text { junc- } \\
\text { tion Toad No. } 2 \text { to } \\
\text { Barceloneta }\end{array}$ & 23 & 6.0 & $\cdots$ & 27.2 & 36.4 & 36.4 & .222 & 52.72 & .024 & .474 \\
\hline Toa clay & $\begin{array}{l}\text { Krn. } 82.3 \text { Cambalache- } \\
\text { Arecibo road }\end{array}$ & 17 & 7.9 & $\cdots$ & 36.4 & 30.8 & 32.8 & .208 & 55.30 & .037 & .321 \\
\hline Tories clay & $\underset{\text { road, Cagtas }}{\mathrm{Km}} \mathbf{0 . 4}$ Borinquen & 500 & 7.5 & 1.39 & 19.2 & 28.8 & 52.0 & .154 & 55.73 & .008 & .204 \\
\hline Yabucoa clay & $\begin{array}{l}\text { About } 34 \mathrm{~lm} \text {, Yabucon- } \\
\text { Calabazas road on } \\
\text { Luis Toro's farm }\end{array}$ & $\cdots$ & 5.2 & 1.17 & 41.6 & 24.4 & 34.0 & .167 & 51.35 & .006 & .163 \\
\hline
\end{tabular}

TARZE 6. AVERAGE VALUES FOR VOLUAIE WEIGHT, WEIGHT OF ACRE PLOW DEPTH, MECHANICAL ANALYSIS, TOTAL AND AVAILABLE PHOSPHORIC ACID AND SILICA AND RATIO OF TOTAL TO AVAILABLE PHOSPHORIC ACID OF ONE HUNDRED NINE SOILS IN THE HUMID REGION OF PUERTO RICO CLASSIFIED INTO FIVE TEXTURAT SOII CEASSES

\begin{tabular}{|c|c|c|c|c|c|c|c|c|c|c|c|}
\hline \multirow{3}{*}{$\begin{array}{l}\text { Number } \\
\text { of } \\
\text { Samples }\end{array}$} & \multirow{3}{*}{$\begin{array}{l}\text { Textural } \\
\text { Soil Class }\end{array}$} & \multirow{3}{*}{$\begin{array}{l}\text { Yolume } \\
\text { Veight }\end{array}$} & \multirow{3}{*}{$\begin{array}{l}\text { Weight of } \\
\text { acre plow } \\
\text { depth } \\
62 / 3 \text { inches, } \\
\text { Ibs. }\end{array}$} & \multicolumn{7}{|c|}{ Percentage on Dry Basis } & \multirow{3}{*}{$\begin{array}{c}\text { Total to } \\
\text { Available } \\
\mathrm{P}_{2} \mathrm{O}_{5} \\
\text { ratio }\end{array}$} \\
\hline & & & & \multicolumn{3}{|c|}{ Mechanical Analysis } & \multicolumn{2}{|c|}{ Total } & \multicolumn{2}{|c|}{ Available } & \\
\hline & & & & Sand & Silt & Clay & $\mathrm{P}_{2} \mathrm{O}_{5}$ & $\mathrm{SiO}_{2}$ & $\mathrm{P}_{2} \mathrm{O}_{5}$ & $\overline{\mathrm{SiO}_{2}}$ & \\
\hline $\begin{array}{r}3 \\
19 \\
20 \\
14 \\
53\end{array}$ & $\begin{array}{l}\text { Sands } \\
\text { Sandy loams } \\
\text { Sandy clay Ioa } \\
\text { Clay loams } \\
\text { Clays }\end{array}$ & $\begin{array}{l}\dddot{3} \dot{4}^{*} \\
1.33 \\
1.31 \\
1.23\end{array}$ & $\begin{array}{r}2,159,414^{*} \\
.2,008,406 \\
1,978,205 \\
1,857,398\end{array}$ & $\begin{array}{l}82.73 \\
68.71 \\
55.32 \\
43.60 \\
34.12\end{array}$ & $\begin{array}{r}2.87 \\
16.57 \\
20.86 \\
29.67 \\
26.68\end{array}$ & & $\begin{array}{l}.051 \\
.106 \\
.125 \\
.116 \\
.140\end{array}$ & & $\begin{array}{l}.002 \\
.017 \\
.009 \\
.007 \\
.007\end{array}$ & $\begin{array}{l}.021 \\
.114 \\
.087 \\
.165 \\
.154\end{array}$ & $\begin{array}{r}22.2 \\
6.3 \\
13.9 \\
16.6 \\
20.0\end{array}$ \\
\hline
\end{tabular}

* Not determined. 


\section{SUMMARY}

One hundred and nine soil types from the humid region of Puerto Rico were classified on the basis of their mechanical analysis into five soil classes - sands, sandy loams, sandy clay loams, clay loams, clays - and were analyzed for $\mathrm{pH}$, volume weight, total and available phosphoric acid and silica. The clay loams weigh about two million pounds per acre plow depth of $6 \frac{2}{3}$ inches, the sandy loams weigh about $5 \%$ more and the clays weigh about $10 \%$ less. The total phosphoric acid varies from $.051 \%$ in the sands to $.140 \%$ in the clays. The available phosphoric acid varies from $.007 \%$ in the clays to $.017 \%$ in the sandy loams. The total to available $\mathrm{P}_{2} \mathrm{O}_{5}$ ratio varies from 6.3 in the sandy loans to 20.0 in the clays. The total silica varies from $56.75 \%$ in the clays to $82.96 \%$ in the sands. The available silica varies from $.021 \%$ in the sands to $.166 \%$ in the clay loams.

\section{LITERATURE CITED}

1. Bonmet, J. A, and Villamil, F. A. Research on shortening time without affecting the accuracy of Dyer's modified method for determination in soils, of phosphoric acid, lime and potash soluble in citric acid solution. Jour. Dept. Agr. Puerto Rico 17(4) : 287-300. 1933.

2. Bouyoucos, G. J. A comparison between the pipette method and the hydrometer method for making mechanical analyses of soị. Soil Sc, 38(5): 335-343. 1934.

3. Curry, A. S. A comparison of methods for determining the volume weight of soils. Jour. Agr. Res, 42(11) : 765-772. 1931.

4. Davis, R. O. E. and Bennett, H. H. Grouping of soils on the basis of mechanical analysis. U. S. D. A. Dept. Circ. 419: 1-14. 1927.

\section{SUMARIO}

Ciento nueve tipos de suelos en la zona húmeda de Puerto Rico se clasificaron a base del análisis mecánico en cinco clases đe suelos: arenas, arenosolómicas, arenoarcillosolómicas, arcillosolómicas, arcillas, y se les hicieron determinaciones de $\mathrm{pH}$, peso por volumen, ácido fosfórico y sílica total y asimilable. El promedio de los valores indica que las clases arcillosolómicas pesan cerca de dos millones de libras por acre arable ( $6 \%$ pulgadas de espesor), las arenosolómicas pesan como $5 \%$ más y las arcillas pesan coma $10 \%$ menos; el ácido fosfórico total varía desde $.051 \%$ en les arenas a $.140 \%$ en las arcillas; el ácido fosfórico asimilable varía desde $.007 \%$ en las arcillas a $.017 \%$ en las clases arenosolómicas; la proporción de ácido fosfórico total a asimilable varía desde 6.3 en las clases arenosolómicas a 20.0 en las arcillas; la silica total varía desde $56.75 \%$ en las arcillas a $82.96 \%$ en las arenas; la sílica asimilable varía desde $.021 \%$ en las arenas a $.166 \%$ en las clases arcillosolómicas. 